\title{
The effect of electrical conductivity on nanosecond discharges in distilled water and in methanol with argon bubbles
}

\author{
Ahmad Hamdan ${ }^{1}$, Kristiāns Čerṇevičs ${ }^{1,2}$, and Min Suk Cha ${ }^{1}$ \\ ${ }^{1}$ King Abdullah University of Science and Technology (KAUST), Clean Combustion \\ Research Center (CCRC) and Physical Science and Engineering Division (PSE), Thuwal \\ 23955, Saudi Arabia \\ ${ }^{2}$ School of Chemistry, University of Glasgow, Joseph Black Building, Glasgow G12 8QQ, \\ UK
}

PACS numbers: 52.80. Wq Discharge in liquids and solids; 52.30.-q Plasma dynamics and flow; 52.80.-s Electric discharges.

Email: ahmad.hamdan@kaust.edu.sa ; min.cha@kaust.edu.sa

Keywords: nanosecond discharge, electrical conductivity, discharge in liquids, discharge in bubbles, nanosecond imaging. 


\begin{abstract}
We investigated the effect of a liquid's electrical conductivity (EC) on the physical characteristics of electrical discharges in liquids with gaseous bubbles. Argon gas was supplied into the liquid to form an array of gaseous bubbles in between two electrodes (a pinto-hollow electrode setup). Methanol and water were considered as base liquids, representing a low and a high dielectric permittivity $(\varepsilon)$ liquid respectively, while potassium chloride $(\mathrm{KCl})$ was added to control the EC of the liquids. When increasing the EC of the liquids, we found that the discharge probability was reduced by $46 \%$ for in-water and $38 \%$ for in-methanol discharges. We also found that the injected charge decreased by $\sim 4 \mu \mathrm{C}$ as the EC increased. Moreover, as the gap distance increased from 1 to $2.5 \mathrm{~mm}$, the injected charge decreased by 2 $\mu \mathrm{C}$ for in-water discharge and by $4 \mu \mathrm{C}$ for in-methanol discharge. The plasma emission is another important parameter in characterizing discharges. With increasing the EC, the plasma emission volume decreased linearly by a factor of $\sim 5$. The plasma lifetime was shortened by around $33 \%$ for in-water and $20 \%$ for in-methanol discharges in the case of $d=1 \mathrm{~mm}$, while the decrease was $40 \%$ for in-water and $30 \%$ for in-methanol discharges in the case of $d=2.5$ $\mathrm{mm}$. Using the broadening characteristics of the $\mathrm{H} \alpha$ line, the electron density was estimated during the first $100 \mathrm{~ns}$ by $\sim 3 \times 10^{17} \mathrm{~cm}^{-3}$ for in-water discharges and by $\sim 2 \times 10^{18} \mathrm{~cm}^{-3}$ for inmethanol discharges, and it decreased by about one order of magnitude after $800 \mathrm{~ns}$; note that $n_{e}$ dependence on the EC was not significant. The reported findings provide further understanding of electrical discharges in bubbled liquids and highlight the influence of a liquid's EC, which are useful in the development and optimization of the applications based on such process.
\end{abstract}




\section{INTRODUCTION}

Non-thermal plasmas created by electrical discharges in liquids, which generate chemically active species, UV photons, and shock waves simultaneously, have been attractive alternatives for many applications [1] such as water treatment [2, 3], material synthesis [4, 5], fuel reforming [6,7], and biomedicine [8]. Addition of gaseous bubbles to the liquid has shown that liquid processing is improved because of the decrease of the breakdown voltage and the increase of selectivity of some chemical reactions [7].

Numerous experimental studies have been conducted on electrical discharges in liquids with gaseous bubbles, and various techniques have been applied to fundamentally characterize the physical properties of these discharges. For example, Gershman et al. [9] have used optical spectroscopy to measure the emission of the hydrogen $(\mathrm{H \alpha})$ line and $\mathrm{OH}$ band. Tachibana et al. [10] have shown that the intensities of $\mathrm{H} \alpha$ and $\mathrm{OH}$ are high with $\mathrm{He}$ and $\mathrm{Ne}$ bubbles compared to Ar, $\mathrm{N}_{2}$ or $\mathrm{O}_{2}$ bubbles, attributing these findings to the difference in the electron energy distribution function (EEDF) of the plasma. Bruggeman et al. [11] have found that the breakdown of the electric field increases from 18 to $32.5 \mathrm{kV} / \mathrm{cm}$ as bubble diameter decreases from 0.5 to $0.15 \mathrm{~cm}$. In addition, numerical studies of discharges in bubbled liquids have been addressed by several authors. For example, Babaeva and Kushner [12] have studied the discharge ignition and propagation in air bubbles in liquids with different dielectric permittivity $(\varepsilon)$. Interestingly, they observed that discharge developed surface hugging for high- $\varepsilon$ liquids, while it is developed along bubble axis for low- $\varepsilon$ liquids. The chemistry induced by such discharges has been discussed in computational study by Tian et al. [13] and recently by Levko et al. [14].

The effect of the liquids' electrical conductivity (EC) on discharges without gaseous bubbles has also been addressed by Shih and Locke [15], Sunka et al. [16], and Bruggeman et al. [17]. Shih and Locke [15] studied the generation of $\mathrm{H}_{2} \mathrm{O}_{2}$ and $\mathrm{H}_{2}$ by discharging in aqueous solutions with various ECs. The results showed that the concentration of $\mathrm{OH}$ radicals and $\mathrm{H}_{2} \mathrm{O}_{2}$ decreased with increasing EC. They also observed that the emission intensities of $\mathrm{O}$ and $\mathrm{H}$ radicals and the generation rates of $\mathrm{H}_{2}$ and $\mathrm{O}_{2}$ were optimum at a conductivity of $150 \mu \mathrm{S} / \mathrm{cm}$. A similar conclusion was also provided by Sunka et al. [16] who reported that production of $\mathrm{OH}$ 
and $\mathrm{O}$ radicals was more efficient when water $\mathrm{EC}$ is in the range of $100 \mu \mathrm{S} / \mathrm{cm}$. Bruggeman et al. [17] showed that both the breakdown voltage and the density of electrons are decreased by one order of magnitude as the water EC exceeded $\sim 50 \mu \mathrm{S} / \mathrm{cm}$, while the plasma temperature was unchanged $(\sim 2500-3000 \mathrm{~K})$ as the EC increased from 1 to $700 \mu \mathrm{S} / \mathrm{cm}$.

The effect of both adding gaseous bubbles and changing the EC of the liquid has only been reported in few studies. In the case of water $(\varepsilon=80)$, Shih and Loke [18] have shown that the effect of the water $\mathrm{EC}$ on the chemical generation rates of $\mathrm{H}_{2}$ and $\mathrm{H}_{2} \mathrm{O}_{2}$ was not significant. In the case of low- $\varepsilon$ liquids, Thagard et al. [19] added salt $(\mathrm{NaCl})$ into bubbled liquids (hydrocarbons), and found that electrical breakdown was facilitated. On the other hand, Zhang and Cha [7] and Hamdan and Cha [20] followed another approach by adding distilled water to low- $\varepsilon$ liquids, which led to an increase of the discharge probability because the dielectric constant of the aqueous mixtures had increased. Despite these studies, the influence of electrical conductivity on the physical properties of the electrical discharges under nanosecond excitation in bubbled liquids has not yet been addressed.

In the present study, based on our recent characterization method for nanosecond discharges in small gaseous bubbles (diameter of $\sim 1 \mathrm{~mm}$ ) in distilled water $[21,22]$ and waterheptane mixture [20], we present here the effect of the liquid EC on the discharge characteristics. The liquid EC is controlled by adding $\mathrm{KCl}$, and two liquids are studied, water $(\varepsilon=80)$ and methanol $(\varepsilon=30)$, as typical liquids for high- and low- $\varepsilon$. The discharges were characterized using time-resolved imaging, voltage-current measurement, and optical emission spectroscopy.

\section{EXPERIMENTAL SETUP}

The experimental setup is schematically shown in figure 1. Pin-to-hollow needle electrodes made of stainless steel were vertically installed in a $5 \times 5 \times 10 \mathrm{~cm}$ quartz container, with the gap distance $(d)$ between the electrodes controlled by a micro-positioning device. The upper needle was polished to a sharp tip (radius of curvature $\sim 50 \mu \mathrm{m}$ ) and was connected to a nanosecond-pulsed power supply (FID, FPG-25-15NM), which provided up to $15 \mathrm{kV}$ with a fullwidth at half maximum of $10 \mathrm{~ns}$ in single-shot operating mode. The lower hollow needle with an 
inner- and outer-diameter of 0.3 and $0.5 \mathrm{~mm}$, respectively, was grounded. A high-voltage probe (Tektronix, P6015A) and a current monitor (Pearson, 6585) measured respectively voltage and current waveforms in conjunction with an oscilloscope (Tektronix, DPO-4104B). A mass flow controller introduced argon gas (purity of 99.99\%) into the container through the lower hollow needle, forming gaseous bubbles inside the liquid. The gas flow was maintained at $3 \mathrm{~cm}^{3} / \mathrm{min}$ for $d=1 \mathrm{~mm}$ and $9 \mathrm{~cm}^{3} / \mathrm{min}$ for $d=2.5 \mathrm{~mm}$, which resulted in a vertically aligned array of bubbles with diameters of approximately $1 \mathrm{~mm}$. Different flowrates were used to optimize the discharge probability (DP) for both gaps - as the optimal flowrate for $d=1 \mathrm{~mm}$ was too small for $d=2.5$ $\mathrm{mm}$ - and to ignite the discharges, due to relatively longer distance between two consecutive bubbles. The power supply continuously produced high voltage pulses at $1 \mathrm{~Hz}$ and the steady gas flow to the hollow needle (cathode) generated a train of bubbles. Thus, the size, shape, and position of a bubble, when a high voltage pulse was applied to the anode, could not be predetermined, and only stochastic approach allows us to characterize the observation. An intensified charge-coupled device (ICCD) camera (Princeton Instrument, PI-MAX2) was used to acquire the image of discharge. Two distinct gate times of the ICCD camera were selected: $1 \mu \mathrm{s}$ to capture an overall light emission for a single discharge event, and $2 \mathrm{~ns}$ to analyze the lifetime of the plasma. We placed a light-emitting diode (LED) lamp (Luminus, SST-90) across the container from the ICCD camera for a backlight illumination, so that an interface between the liquid and the bubble could be easily identified. A delay generator (BNC, 575) triggered and synchronized the LED lamp, the ICCD camera, and the high-voltage power supply.
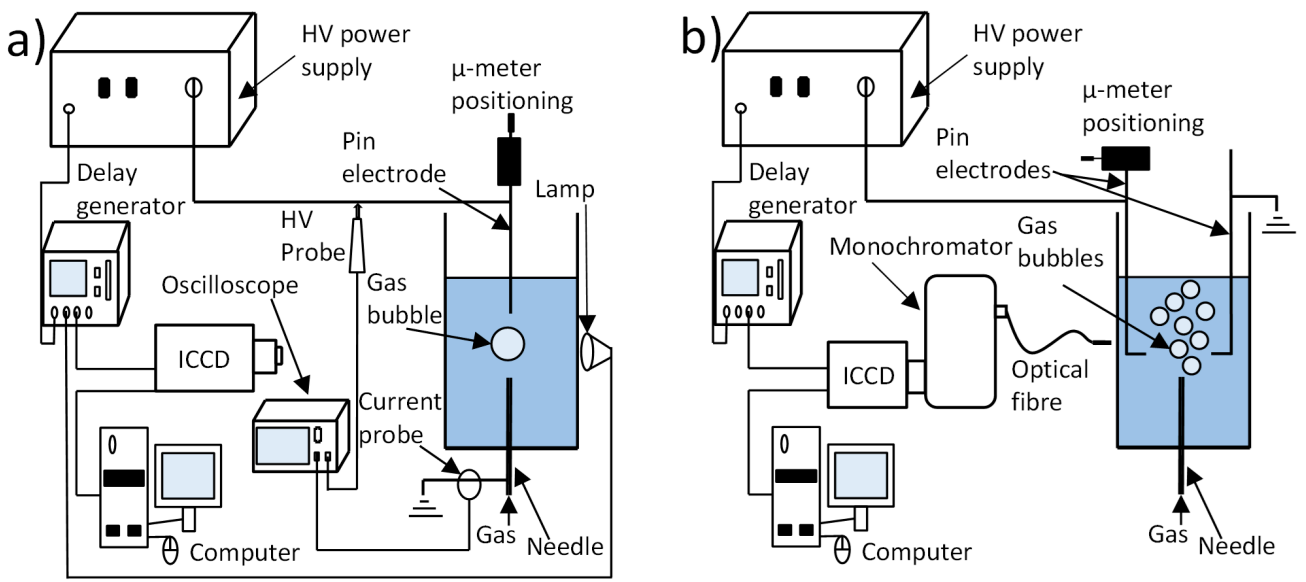

Figure 1. The experimental setup a) for imaging and electrical characterization and b) for optical emission spectroscopy. 
The container was filled with $100 \mathrm{~cm}^{3}$ of liquid in order to submerge the electrode. We used water $(\varepsilon=80)$ and methanol $(\varepsilon=30)$ as base liquids to investigate the dependence of discharge characteristics on $\varepsilon$. Stock solutions with high conductivity $(5 \mathrm{mS} / \mathrm{cm})$ were prepared by adding $\mathrm{KCl}$ into distilled water $(10 \mu \mathrm{S} / \mathrm{cm})$ or methanol $(10 \mu \mathrm{S} / \mathrm{cm})$ and sonification ensured the uniformity of the solutions. The stock solutions were respectively added into distilled water and methanol to obtain a range of conductivity from $10 \mu \mathrm{S} / \mathrm{cm}$ up to $1 \mathrm{mS} / \mathrm{cm}$ in water and up to $2.1 \mathrm{mS} / \mathrm{cm}$ in methanol. Beyond the upper limit of each conductivity range, we were unable to create discharges. The conductivity of each solution was measured using a conductivity/ion/pH meter (Horiba LAQUA F-74).

To obtain reasonably clear light emission spectra, the material and configuration of the electrodes were modified (figure 1b). The stainless steel electrodes had caused emission lines that interfered with the spectra from the gas and liquid substances. These were replaced with two horizontally mounted tungsten electrodes (99.95\% purity). The lower hollow needle was still used to supply bubbles into liquid, but to avoid discharges between the needle and the tungsten electrodes, they were located $1 \mathrm{~cm}$ above the needle. For emission spectroscopy, we used a monochromator (Princeton Instruments, SP2750) equipped with a grating (900 grooves/mm, blazed at visible) and an ICCD camera (Princeton Instrument, PI-MAX3) as a detector. The length of the spectrometer was $750 \mathrm{~mm}$ with entrance slit width of $50 \mu \mathrm{m}$, resulting in a resolution of $0.036 \mathrm{~nm}$ in wavelength. To increase the signal-to-noise ratio, 200 discharges were accumulated and averaged.

\section{RESULTS AND DISCUSSION}

\subsection{Effects of electric conductivity on discharge probability}

We first investigated the effect of the EC on the probability of electrical discharges in water. A discharge probability (DP), defined as the amount of successful discharges out of 200 applied pulses, was introduced to investigate the effect of EC on the discharge for a given voltage and geometrical condition. Note that a successful discharge was determined based on light detection in the ICCD image. The variation of DP in water as a function of EC for two gap 
distances, $d=1$ and $2.5 \mathrm{~mm}$ at $V_{a}=15 \mathrm{kV}$, is shown in figure 2, while a comparison of DP for water $(\varepsilon=80)$ and methanol $(\varepsilon=30)$, for $d=2.5 \mathrm{~mm}$ and $V_{a}=15 \mathrm{kV}$, is shown in figure 3 .

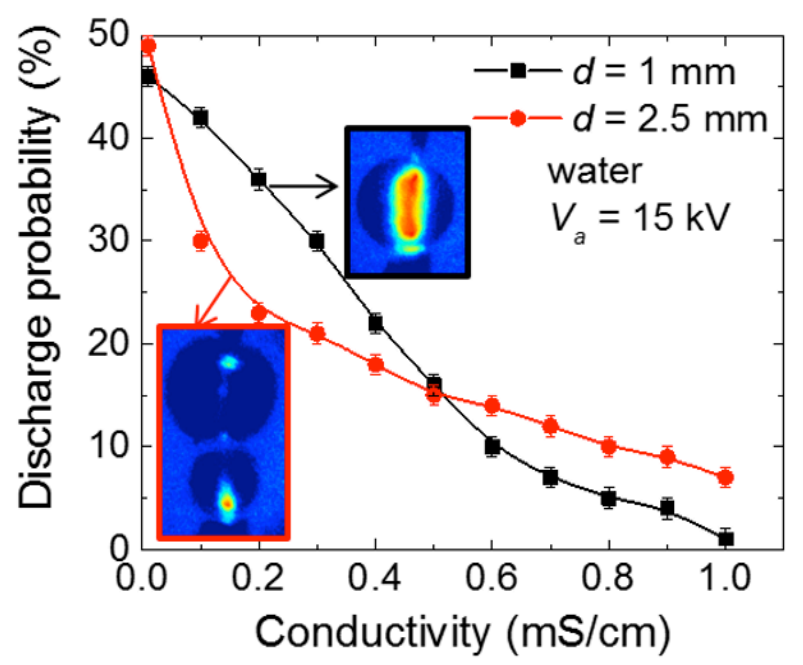

Figure 2. Variation of the discharge probability in water discharge as a function of EC for $d=1$ and $2.5 \mathrm{~mm}$ at $V_{a}=15 \mathrm{kV}$.

The discharge probability decreased with increasing water EC in general. Even for the larger gap $(d=2.5 \mathrm{~mm})$, DP at the smallest EC showed a similar value to the smaller gap of approximately $46-50 \%$ under the same applied voltage. This similarity is counter-intuitive because a representative field intensity between two electrodes can be inversely proportional to the gap distance. This counter-intuitive result can be attributed to the two bubbles found in the larger gap, because a local enhancement of field intensity in the region of bubble-bubble interface facilitates electrical discharges. In the case of $d=1 \mathrm{~mm}$, only one bubble fit in the gap and the discharge could be ignited from the anode, the cathode or from both electrodes [22]. However, for $d=2.5 \mathrm{~mm}$, two bubbles fit in the gap, and the discharge ignition was controlled by their relative position [22]. Thus, the main difference between $d=1$ and $\mathrm{d}=2.5 \mathrm{~mm}$ was the thickness of the liquid layer between the electrodes and the bubble (case of $d=1 \mathrm{~mm}$, see the inset image) and between the inter-bubbles (case of $d=2.5 \mathrm{~mm}$, see the inset). Although both gap distances resulted in comparable ranges of DP for the tested range of EC, a cross-over point can be found in figure 2 at $\sim 0.5 \mathrm{mS} / \mathrm{cm}$ : for $\mathrm{EC}<0.5 \mathrm{mS} / \mathrm{cm}$, the smaller gap showed greater DP, while it resulted in less DP for EC $>0.5 \mathrm{mS} / \mathrm{cm}$. This might be attributed to a combined effect of EC and bubble-bubble interaction on local field intensity to the most probable location 
of electrical discharges (see Supplementary Material for more details about the discharge modes and their current-voltage waveforms (Supplementary figure 1) and their relative percentage distribution (Supplementary figure 2)), but it is difficult to explain experimentally with a detailed mechanism at the moment.

To investigate the effect of the dielectric property of a liquid, water $(\varepsilon=80)$ was replaced by methanol $(\varepsilon=30)$. As shown in figure 3 , for $\mathrm{EC}<0.9 \mathrm{mS} / \mathrm{cm}$, DP in low- $\varepsilon$ liquid (methanol) is smaller than DP in high- $\varepsilon$ liquid (water), as expected from previous studies [7, 20, 23]. For instance, at $\mathrm{EC}=10 \mu \mathrm{S} / \mathrm{cm}$ (the smallest EC), DP was $\sim 50 \%$ in water while it was $\sim 25 \%$ in methanol. However, DP for both liquids showed similar values of DP $\sim 10 \%$ at EC of $0.9 \mathrm{mS} / \mathrm{cm}$, and similar decreasing trends for both liquids can be expected for EC $>0.9 \mathrm{mS} / \mathrm{cm}$. Thus, at higher EC the effect of $\varepsilon$ can be neglected, and so EC is a controlling parameter in discharges, at least for the tested liquids with bubbles. Note that the effect of $\varepsilon$ could not be verified for lower $\varepsilon$ liquids such as $n$-heptane $(\varepsilon=2)$, because $\mathrm{KCl}$ cannot be dissolved in such a non-polar liquid. However, since the DP behavior with $n$-heptane using the same experimental setup [20] showed similar trend of DP as a function of $V_{a}$ to that with distilled water, it can be expected for low $\varepsilon$ liquids to show similar effect of EC on DP as water showed in this study, say deteriorated DP with increased EC.

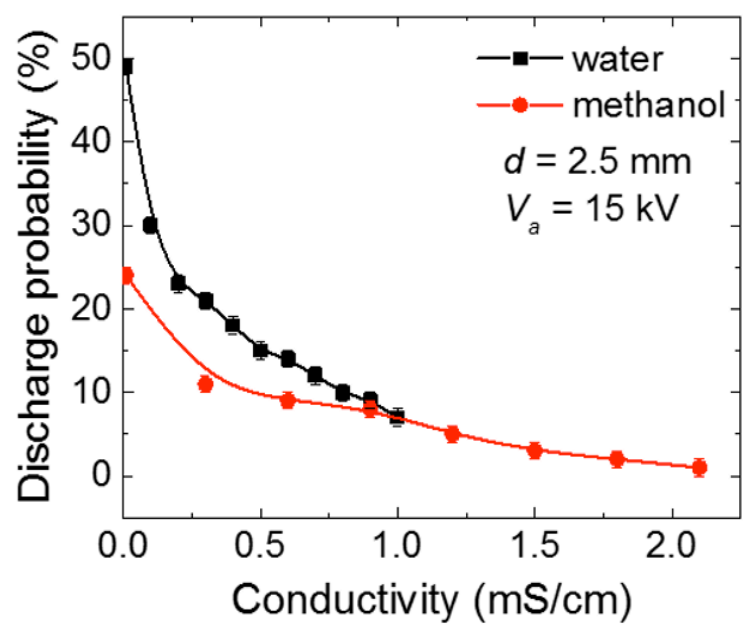

Figure 3. Discharge probability as a function of electric conductivity in water $(\varepsilon=80)$ and methanol $(\varepsilon=30)$ with $d=2.5 \mathrm{~mm}$ applying $V_{a}=15 \mathrm{kV}$. 
The decrease in DP with increased EC can be attributed to the conductive dissipation of the input power by the rapid dissipation of charges accumulated in the medium, such that there is not enough power left to create discharges for high EC. This hypothesis is further supported by the current profiles of misignited discharges (no discharge observed), where higher current flow can be detected for higher EC of the liquids. Figures $4 \mathrm{a}$ and $4 \mathrm{~b}$ present the current profiles of misignited events for selected EC in methanol and water, respectively. During the first $10 \mathrm{~ns}$, the current $\left(i_{C}\right)$ does not depend on the EC, indicating that the capacitive components $(C)$ are due to the time increase of the voltage $\left(i_{C}=C d V_{a} / d t\right)$. Comparing water to methanol, one can deduce that the equivalent capacitance in methanol (current peak value $\sim 25 \mathrm{~A}$, figure $4 \mathrm{a}$ ) is higher than in water (current peak value $\sim 15 \mathrm{~A}$, figure $4 \mathrm{~b}$ ). On the other hand, beyond $10 \mathrm{~ns}$, the effect of EC on the current waveform is clear: as the liquid EC increases, the current increases for both liquids, water and methanol.
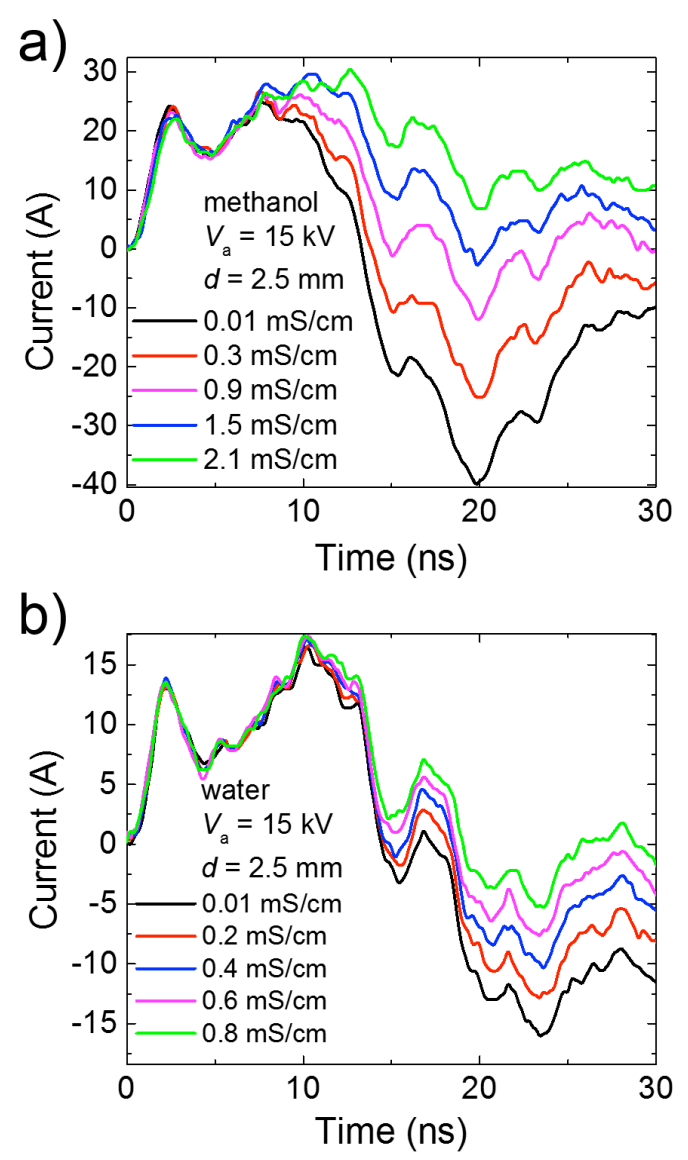

Figure 4. Current profiles of the misignited discharges for methanol (a) and water (b) solutions of various ECs with $d=2.5 \mathrm{~mm}$ applying $V_{a}=15 \mathrm{kV}$. 


\subsection{Characterization of discharges}

The influence of liquid EC on the spatial distribution of the plasma emission was investigated using the ICCD camera. Figure 5 shows typical images of emission from discharges in-water and in-methanol for $d=1 \mathrm{~mm}$ and $V_{a}=15 \mathrm{kV}$. For the in-water discharges, the plasma emission moves from a homogenous emission column for the smallest EC $(10 \mu \mathrm{S} / \mathrm{cm})$, to become localized near the electrodes with increased EC, while the in-methanol discharges result in rather homogeneous emission along the discharge axis showing negligible effect of EC. In a previous study [22], the co-ignition mode of discharge in distilled water was reported, in which two ignition kernels at the anode and the cathode simultaneously start to grow (during the first $\sim 50 \mathrm{~ns}$ ) and expand axially, then connect with each other, forming a plasma channel (from 50 to $200 \mathrm{~ns}$ ). For the in-water discharges, a uniformly connected discharge column can be found only at the lowest EC (figure 5,10 $\mu \mathrm{S} / \mathrm{cm}$ ), while the connection between two kernels becomes weaker as the EC increases (figure 5, 0.3 and $1 \mathrm{mS} / \mathrm{cm}$ ). However, the emission characteristics are significantly different for the in-methanol discharges. Although the volume of emission seems to be slightly decreased with increased EC, the solid formation of uniform plasma channels is rather unaffected by EC.

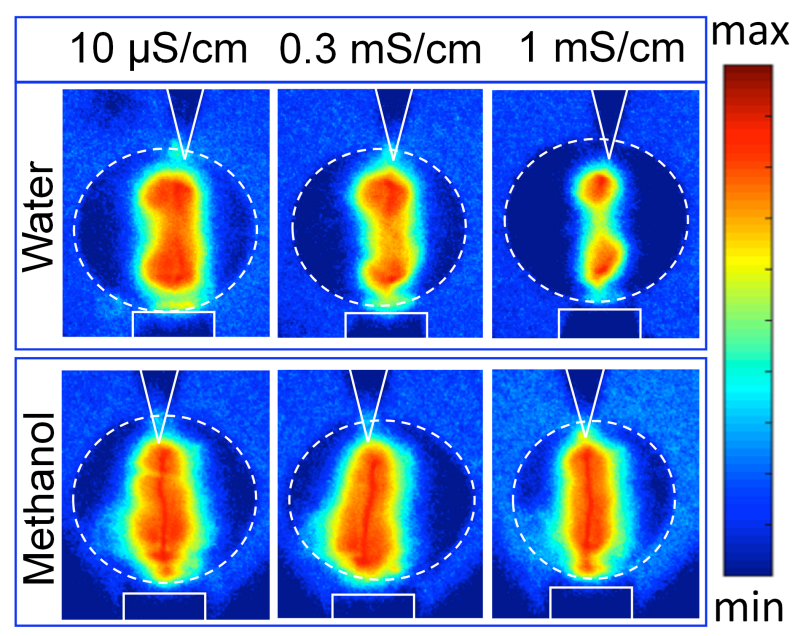

Figure 5. Images (exposure time $=1 \mu \mathrm{s}$ ) of discharges for various EC in water and methanol with $d=1 \mathrm{~mm}$ applying $V_{a}=15 \mathrm{kV}$. 
To further understand discharge characteristics, we estimated the volume of the emission zone of the discharge. Assuming the discharge columns consist of finite number of circular disk having a height of 1 pixel length, the total volume of the emission zone can be estimated as a sum of the volume of each elementary disk. The number of elementary disks is the number of pixels in the vertical direction (Supplementary figure 3). By taking a certain intensity detected as a threshold to indicate the minimum value for the active plasma zone, we could determine the width of the discharge column at a specific vertical location (pixel), which can be treated as a diameter of the corresponding disk. Then, by converting the number of pixels in a physical dimension ( $5 \mu \mathrm{m} / \mathrm{pixel})$, we could estimate the volume of each elementary disk. The variation in the emission volume with increased conductivity for both in-water and in-methanol discharges is presented in figure 6 for $d=1 \mathrm{~mm}$ and $V_{a}=15 \mathrm{kV}$. The emission volume decreased linearly with EC, exhibiting a more rapid decrease for the case of in-water discharge as compared to inmethanol discharge.

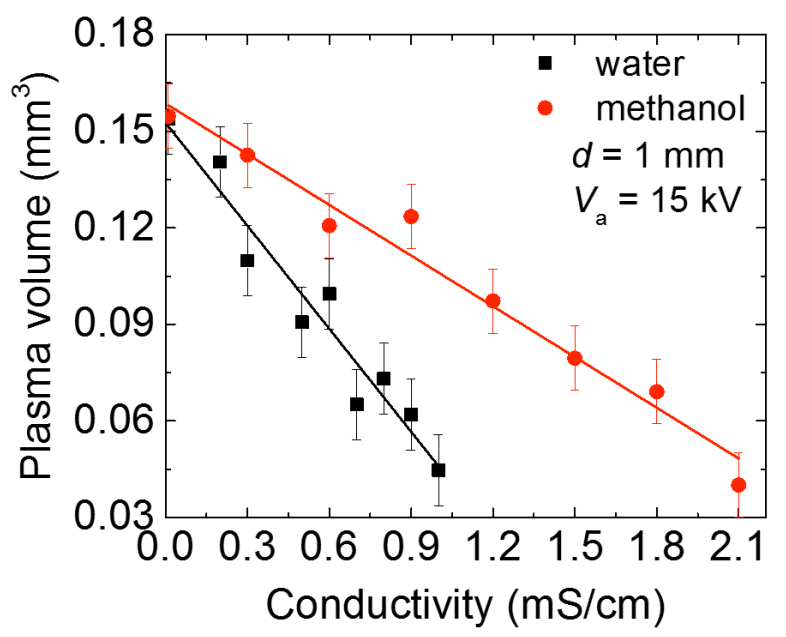

Figure 6. The variation of the emission volume as a function of EC of water and methanol with d $=1 \mathrm{~mm}$ and $V_{a}=15 \mathrm{kV}$.

The lifetime of the plasma emission is another important parameter in characterizing discharges. To determine the emission lifetime, the exposure time of the ICCD camera was narrowed down to $2 \mathrm{~ns}$ and a time delay was induced to find a starting and ending moment (a time window) of light emission. Figure 7 shows the experimentally determined lifetimes of light emission for the in-water and in-methanol discharges with two inter-electrodes gaps $(d=1$ and 
$2.5 \mathrm{~mm})$. In general, increases in EC resulted in shorter lifetimes of light emission. For $d=1$ $\mathrm{mm}$, the lifetime of light emission of the in-water discharge was $\sim 1.8 \mu \mathrm{s}$ at EC of $10 \mu \mathrm{S} / \mathrm{cm}$ and it decreased linearly to $\sim 1.2 \mu \mathrm{s}$ at EC of $1 \mathrm{mS} / \mathrm{cm}$. However, the lifetimes for the in-methanol discharges were higher than those for in-water discharges: they were $\sim 2.2 \mu \mathrm{s}$ at EC of $10 \mu \mathrm{S} / \mathrm{cm}$ and decreased linearly to $\sim 1.8 \mu \mathrm{s}$ at EC of $2.2 \mathrm{mS} / \mathrm{cm}$. As illustrated in figure 7, for $d=2.5 \mathrm{~mm}$, the lifetime for each liquid shows a similar decreasing behavior with EC exhibiting shorter lifetime in value as compared to the cases with $d=1 \mathrm{~mm}$.

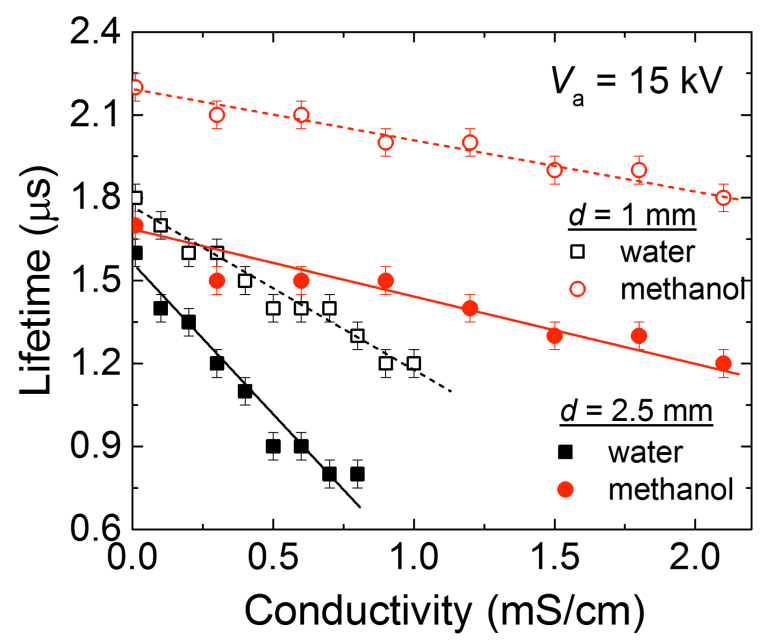

Figure 7. Lifetime of the plasma emission with increasing EC of water and methanol with $V_{a}=$ $15 \mathrm{kV}$ and for $\mathrm{d}=1$ and $2.5 \mathrm{~mm}$.

Because the injected charge $\left(Q_{\text {injected }}\right)$ can be related to some properties of the discharge, such as an electron density [21], its behavior as a function of EC was studied. To determine

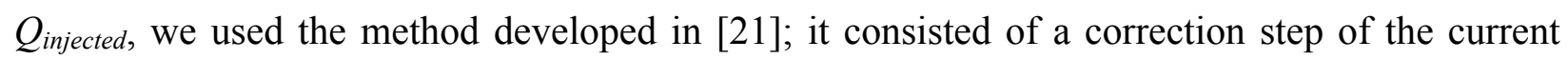
waveforms using that of the misignited waveforms. This procedure allowed us to suppress the current components from displacement current, including system-dependent currents due to electrical connections and supplemental electric devices. Then, $Q_{\text {injected }}$ is calculated by time integration of the corrected current waveform. Figure 8 illustrates the calculated $Q_{\text {injected }}$ along increased EC for in-water and in-methanol discharges at $d=1$ and $2.5 \mathrm{~mm}$ applying $V_{a}=15 \mathrm{kV}$. $Q_{\text {injected }}$ depends on the gap, liquid, and EC. When the gap has reduced from 2.5 to $1 \mathrm{~mm}, Q_{\text {injected }}$ increases by $\sim 4 \mu \mathrm{C}$ for in-methanol discharges and by $\sim 2 \mu \mathrm{C}$ for in-water discharges over the whole range of tested EC. The reduction of $Q_{\text {injected }}$ by increasing the gap is a common 
phenomenon that we observed previously [20,21], and it has been reported to the loss of charged species as they traveled longer distance. For in-water discharges, $Q_{\text {injected }}$ decreases linearly with EC; for instance, at $d=1 \mathrm{~mm}, Q_{\text {injected }}$ decreases by $\sim 4 \mu \mathrm{C}$ as the EC increases from 0.01 to 1 $\mathrm{mS} / \mathrm{cm}$, while at $d=2 \mathrm{~mm}, Q_{\text {injected }}$ decreases by $\sim 2 \mu \mathrm{C}$ as the EC increases from 0.01 to 0.5 $\mathrm{mS} / \mathrm{cm}$. However, for in-methanol discharges, the variation of $Q_{\text {injected }}$ as a function of EC is different because two slopes have been identified. For EC ranging from 0.01 to $0.6 \mathrm{mS} / \mathrm{cm}$, the decrease of $Q_{\text {injected }}$ is similar to in-water discharge; for instance, it decreases by $\sim 2 \mu \mathrm{C}$ for both gaps, $d=1$ and $2.5 \mathrm{~mm}$. However, for $0.6<\mathrm{EC}<2.1 \mathrm{mS} / \mathrm{cm}$, the variation of $Q_{\text {injected }}$ is slow; it only decreases by $\sim 0.5 \mu \mathrm{C}$ for $d=1 \mathrm{~mm}$ and by $1 \mu \mathrm{C}$ for $d=2.5 \mathrm{~mm}$.

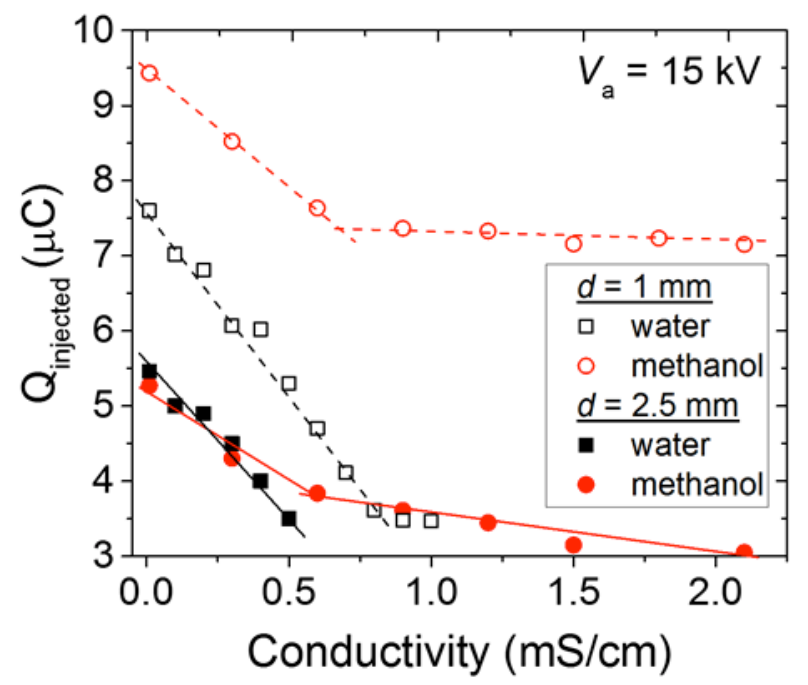

Figure 8. The variation of the $Q_{\text {injected }}$ as a function of EC of in-water and in-methanol discharges with $V_{a}=15 \mathrm{kV}$ and for $d=1$ and $2.5 \mathrm{~mm}$.

To obtain quantitative information on the in-liquid discharges under the various EC, optical emission spectroscopy was adopted with the modified setup (see section 2, figure 1b). The measured spectral range was between 300 and $800 \mathrm{~nm}$. Figure 9 shows the emission spectra for the in-methanol discharges with selected EC; spectrum of water with EC of $10 \mu \mathrm{S} / \mathrm{cm}$ is superimposed on those spectra. As EC increased, individual spectral lines for specific species as well as continuous background emission decreased gradually. Note that the global emission intensity for in-water discharges was small compared with in-methanol discharges, at similar conditions of gap, EC, and voltage. This difference in intensities can also be observed from the ICCD images (see figure 5 at $\mathrm{EC}$ of $10 \mu \mathrm{S} / \mathrm{cm}$ ), where an intense thin channel can be observed in 
the middle of the plasma channel. This can explain the relatively high intensity for in-methanol discharges compared to the in-water discharges, although the plasma volume is comparable.

The main peaks were identified as $\mathrm{H} \alpha, \mathrm{H} \beta, \mathrm{CH}, \mathrm{C}_{2}$, and $\mathrm{O}$ in the spectrum, while the fine structure of smaller peaks was due to $\mathrm{Ar}_{\mathrm{I}}, \mathrm{O}_{\mathrm{I}}, \mathrm{H}_{\mathrm{I}}$, and $\mathrm{W}_{\mathrm{I}-\mathrm{II}}$; second order of $\mathrm{W}_{\mathrm{I}-\mathrm{II}}$ was also observed as shown in the inset of figure 9. Tungsten peaks were resulting from the electrode erosion and their intensity was more significant at low EC; this was caused by higher discharge power with low EC. $\mathrm{Ar}_{\mathrm{I}}$ originated from the supplied gas, while $\mathrm{O}_{\mathrm{I}}$ and $\mathrm{H}_{\mathrm{I}}$ resulted from the dissociation of methanol. Interestingly, we did not observe $\mathrm{K}$ or $\mathrm{Cl}$ lines in the spectra, indicating that these species did not diffuse into the gaseous bubble, keeping EC in the bubble unchanged. The continuum observed in the spectra (more pronounced for lower EC) was probably due to electron-ion recombination, which is in agreement with the high volume, $Q_{\text {injected }}$, and lifetime of the plasma emission at low EC.

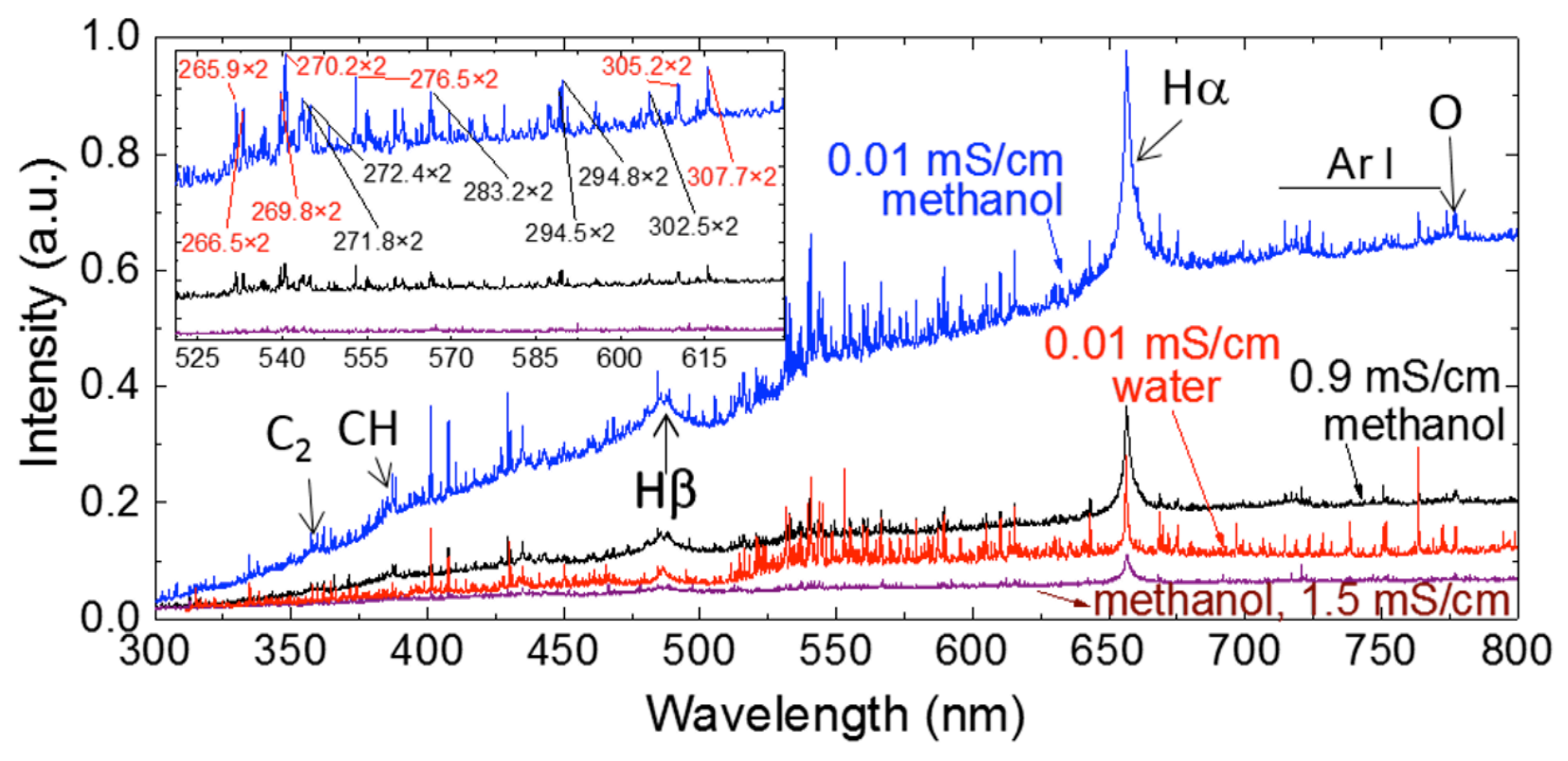

Figure 9. Emission spectra obtained by discharging in methanol ( $E C=0.01,0.9$, and 1.5 $\mathrm{mS} / \mathrm{cm})$ and in water $(E C=0.01 \mathrm{mS} / \mathrm{cm})$ with $d=1 \mathrm{~mm}$ and $V_{a}=15 \mathrm{kV}$; the exposure time is 1 $\mu$ s. The insert is a zoom in 520-630 $\mathrm{nm}$ region showing the second order of $W_{I}$ (black) and $W_{I I}$ (red) lines.

We finally used the profile of the hydrogen line $(\mathrm{H} \alpha)$ to calculate and discuss the temporal evolution of the spatial-averaged electron densities $\left(n_{e}\right)$ in plasma. Assuming that the 
line broadening is mainly due to Stark broadening, the profile can be fitted by the Lorentzian profile [21]. Thus, $n_{e}\left(\mathrm{~cm}^{-3}\right)$ is linked to the full-width at half maximum $\Delta \lambda_{\mathrm{FWHM}}(\mathrm{nm})$ of the Lorentzian profile by:

$$
n_{\mathrm{e}}=8.8308 \times 10^{16}\left(\Delta \lambda_{\mathrm{FWHM}}\right)^{1.6005}
$$

Typical peaks of $\mathrm{H} \alpha$, obtained by discharging in water and methanol, at $d=2.5 \mathrm{~mm}, V_{a}=$ $15 \mathrm{kV}$, and $\mathrm{EC}=10 \mu \mathrm{S} / \mathrm{cm}$, are presented in figure $10 \mathrm{a}$ and $10 \mathrm{~b}$, respectively. The $\mathrm{H} \alpha$ lines clearly show different broadenings indicating different electron densities, especially during the first 200 ns: in-water discharges $n_{e} \sim 2-3 \times 10^{17} \mathrm{~cm}^{-3}$ while in-methanol discharges $n_{e} \sim 1-2 \times 10^{18}$ $\mathrm{cm}^{-3} \cdot n_{e}$ decreases with the time and it drops by about one order of magnitude after $1 \mu \mathrm{s}$ in both liquids (figure 10c). The decrease of $d$ from 2.5 to $1 \mathrm{~mm}$ shows close values of $n_{e}$, indicating weak dependence between $d$ and $n_{e}$. Note that the $n_{e}$ calculated in-methanol discharge is higher than in-water discharge along the whole discharge time. Also, the variation of $n_{e}$ as a function of EC is not significant in both liquids. For the in-water discharges, the order of $n_{e}$ is in good agreement with that reported in literature [24, 25]; however, for the in-methanol discharges, present estimation of $n_{e}$ is relatively high compared to the reported data [12]. Concerning the effect of EC on the electron density, Vanraes et al. [24] also reported that $n_{e}$ is almost independent of EC, although their reported electron density based on $\mathrm{H \alpha}$ for $5 \mathrm{mS} / \mathrm{cm}$ is slightly smaller than that of distilled water. It should be noted that the in-water discharges without gaseous bubbles have shown strong dependence of $n_{e}$ on EC [17].
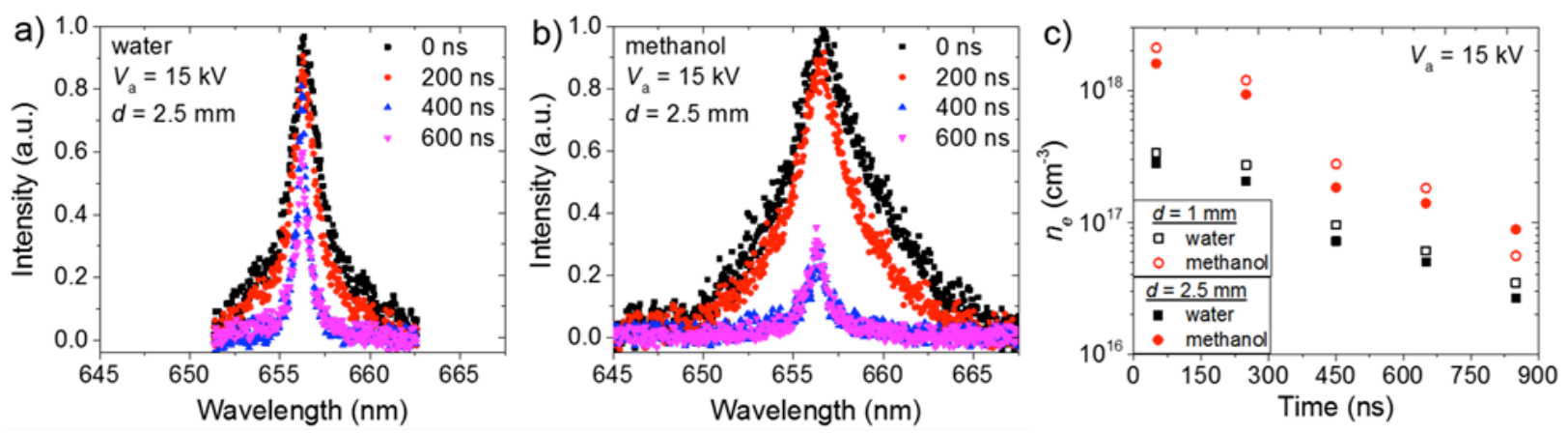

Figure 10. Time evolution of Ha lines observed by discharging in a) water and b) methanol with $d=2.5 \mathrm{~mm}, V_{a}=15 \mathrm{kV}$, and EC of $10 \mu \mathrm{S} / \mathrm{cm}$; the exposure time is $100 \mathrm{~ns}$. c) Time evolution of $n_{e}$ for water and methanol and for $d=1$ and $2.5 \mathrm{~mm}$ at $V_{a}=15 \mathrm{kV}$. 
Finally, we needed to address the higher electron density with the low- $\varepsilon$ liquid (methanol), which was an unexpected observation based on the simulations presented by Babaeva and Kushner [12], where $n_{e}$ in water $\left(8 \times 10^{16} \mathrm{~cm}^{-3}\right)$ was slightly higher than that in methanol $\left(7 \times 10^{16} \mathrm{~cm}^{-3}\right)$. We presumed that the reason was related to the relative size of a gas bubble with respect to the inter-electrode gap distance and to the discharge mode. In the previous simulation [12], the bubble was close to the anode but relatively far from the cathode; in our case the bubble was close to both electrodes, which further increased the intensity of the electric field inside the bubble, especially at the anode- and cathode-pole sides. In addition, during the experiment, we observed a continuous plasma channel between the electrodes together with an emission from the electrode material due to erosion, which supports that the discharge was in spark mode, whereas the discharge is supposed to be in corona mode in the simulation [12].

To verify this fact in the present experimental conditions (bubble diameter $=1 \mathrm{~mm}, d=1$ $\mathrm{mm}, V_{a}=15 \mathrm{kV}$, pin-to-hollow needle electrodes configuration), we simulated the spatial distribution of the static electric field (figure 11a) using a commercial software, Comsol Multiphysics, as the simulation presented in [22]. We found that the local field intensity near the anode as well as at the bubble pole (anode side) was higher with low- $\varepsilon$ liquid $(\varepsilon=30)$ than with high- $\varepsilon$ liquid $(\varepsilon=80)$. In figure $11 \mathrm{~b}$, we co-plotted the profiles of the electric field intensity along the electrode (axisymmetric) axis with two liquids ( $\varepsilon=30$ and 80 ). The color code of the figure represents the spatial distribution of the electric field and its intensity. The electric field intensity of the in-methanol case was higher than that of in-water. The decrease in dielectric permittivity from 80 to 30 resulted in a 10\% increase in the field intensity at the bubble (anode side) and 18\% increase near the anode. This increase in the field intensity may explain the relatively higher $n_{e}$ experimentally observed for the in-methanol discharges compared to the in-water discharges. Such locally enhanced field intensity with low- $\varepsilon$ liquid may also help to explain the greater volume and longer lifetime of light emission with the in-methanol discharges. 


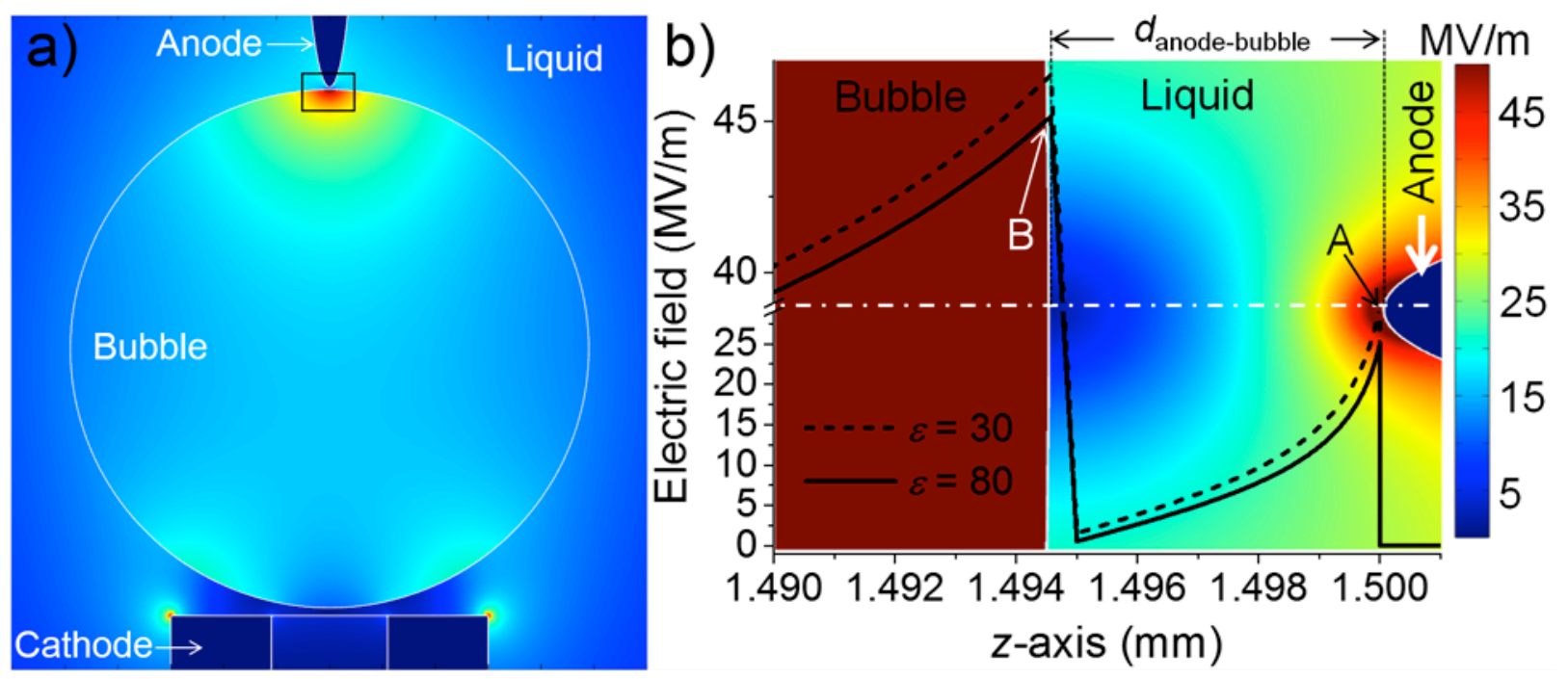

Figure 11. a) Numerical simulations of electric field for $d=1 \mathrm{~mm}$, bubble diameter $=1 \mathrm{~mm}$, and $V_{a}=15 \mathrm{kV}$ in water $(\varepsilon=80)$. b) Zoom of the square drawn in a) and we show the anode tip and the anode-side of the bubble. The background is for $\varepsilon=80$ while the profiles along the bubble axis are for $\varepsilon=80$ (solid line) and 30 (dashed line).

By changing some conditions in the simulation, we investigated the effect of the distance (anode to bubble) and $\varepsilon$ on the intensity of the electric field at the anode and anode-side of the bubble. Consistently, the range of $\varepsilon$ has been chosen from 2 to 80 while the values of anodebubble distance $\left(d_{\text {anode-bubble }}\right)$ are 1,5 , and $10 \mu \mathrm{m}$; the results are presented in figure 12 . First, for low $\varepsilon$, the intensity of the electric field at the anode was higher than that at the anode-side of the bubble. The increase of $\varepsilon$ led to the decrease of the intensity of the electric field at both sides (anode and bubble), but the decrease rate depended on the $d_{\text {anode-bubble. For instance, when } d_{\text {anode- }}}$ bubble $=1 \mu \mathrm{m}$, the intensity of the electric field at the anode decreased from $12 \times 10^{7}$ to $0.6 \times 10^{7}$ $\mathrm{V} / \mathrm{m}$, while at the anode-side bubble, it decreased from $11 \times 10^{7}$ to $5 \times 10^{7} \mathrm{~V} / \mathrm{m}$. At $\varepsilon=2.2$, the intensity of the electric field became equal at both sides. Increasing $d_{\text {anode-bubble }}$ led to an increase of the intensity of the electric field at the anode, while it decreased at the anode-side bubble. Interestingly, for high $\varepsilon$, the intensity of the electric field at the anode-side bubble remained higher than that at the anode. The equality of the electric field at both sides (anode and bubble) was observed for $\varepsilon \sim 2.2,7.8$, and 60 and for $d_{\text {anode-bubble }}$ of 1,5 , and $10 \mu \mathrm{m}$. The reported results showed how the intensity of the electric field and its spatial distribution were sensitive to the anode-bubble distance and $\varepsilon$. From an experimental point of view, it was quite difficult to control 
the anode-bubble position at the micrometer scale, and it seemed to play an important role on the breakdown mechanism, in particular for low- $\varepsilon$ liquids (e.g., $\varepsilon=2$ ) where the intensity of the electric field at the anode-side bubble changes from $\sim 11 \times 10^{7}$ to $\sim 5 \times 10^{7} \mathrm{~V} / \mathrm{m}$ as the anode-bubble distance changes from 1 to $10 \mu \mathrm{m}$ - this change is usually neglected in experiments.
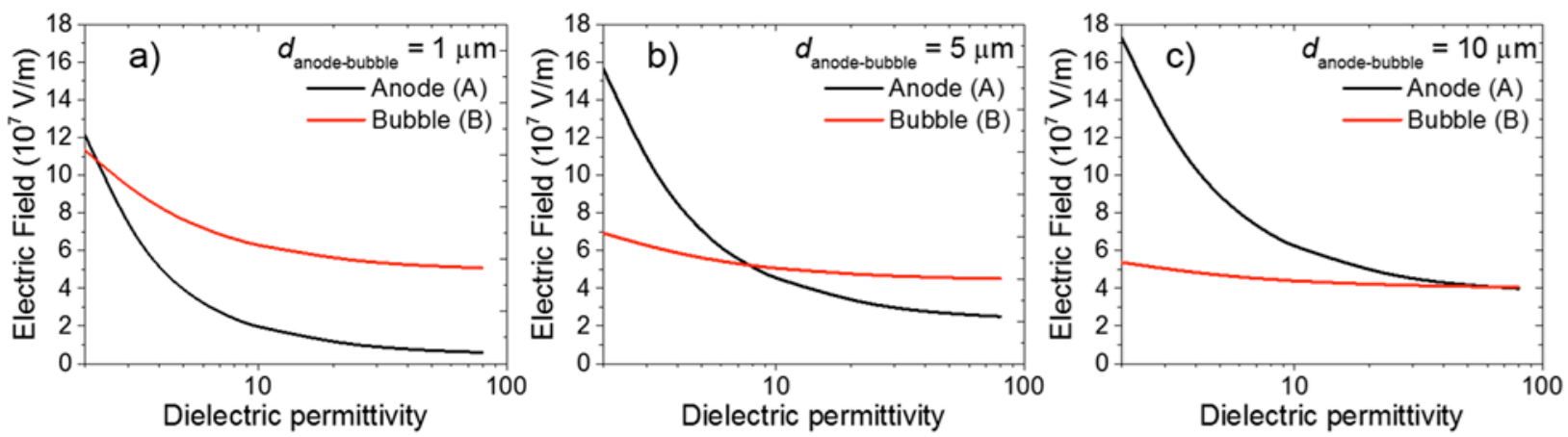

Figure 12. a) Variation of the intensity of electric field at the anode and the anode-side bubble as a function of $\varepsilon$ for anode-bubble distance of a) $1 \mu \mathrm{m}, \mathrm{b}) 5 \mu \mathrm{m}$, and c) $10 \mu \mathrm{m} . \mathrm{d}=1 \mathrm{~mm}$, bubble diameter $=1 \mathrm{~mm}$, and $V_{a}=15 \mathrm{kV}$.

\section{CONCLUSION}

We have investigated the effect of liquid EC on the physical characteristics of nanosecond discharges in bubbled liquids. First, the DP showed a decrease as a function of liquid $\mathrm{EC}$ and the decrease rate depended on $d$ and $\varepsilon$. The volume of the plasma emission as well as its lifetime showed a linear decrease with increasing EC, however, the rate of decrease in water was more pronounced than in methanol. The $Q_{\text {injected }}$ was also determined and it decreased with increasing EC. Although the decrease was linear for both liquids, two slopes were identified for in-methanol discharge and the decrease of $Q_{\text {injected }}$ became slow when the EC exceeded 0.6 $\mu \mathrm{S} / \mathrm{cm}$. Also, increasing $d$ from 1 to $2.5 \mathrm{~mm}$ resulted in decreasing $Q_{\text {injected }}$ by $\sim 2 \mu \mathrm{C}$ for in-water discharges and by $\sim 4 \mu \mathrm{C}$ for in-methanol discharges. Finally, for a given liquid, $n_{e}$ was found constant over the whole range of the EC, although the global emission intensity decreased. By decreasing $\varepsilon$ from 80 to $30, n_{e}$ decreased by a factor of $\sim 10$. This behavior was clarified by simulating the electric field distribution, and we found that the field intensity at the anode and at the bubble pole (anode side) is highest for low- $\varepsilon$ liquids. Our findings provide further understanding of the discharge dynamics in bubbled liquids of various electrical conductivities 
and dielectric permittivities, which is important for the development and optimization of applications such as water treatment and fuel reforming.

\section{Acknowledgement}

The research reported in this publication was supported by Competitive Research Funding from King Abdullah University of Science and Technology (KAUST). 


\section{REFERENCES}

[1] Sun B, Sato M, and Clements J S 1997 Journal of Electrostatics 39(3) 189-202.

[2] Foster J, Sommers B, Gucker S, Blankson I, and Adamovsky G 2012 IEEE Trans Plasma Sci. 40(5) 1311-1323.

[3] Yang Y, Kim H, Starikovskiy A, Fridman A, and Cho Y 2010 Water Research 44(12) 3659-3668.

[4] Vlad I, Marisca O, Vulpoi A, Simon S, Leopold N, and Anghel S 2014 Journal of Nanoparticle Research 16(10) 2633.

[5] Belmonte T, Hamdan A, Kosior F, Noël C, and Henrion G 2014 J. Phys. D: Appl. Phys. 47(22) 224016.

[6] Piavis W, Turn S, and Mousavi A 2015 International Journal of Hydrogen Energy 40(39) 13295-13305.

[7] Zhang X and Cha M S 2015 J. Phys. D: Appl. Phys. 48215201.

[8] van Gils C, Hofmann S, Boekema B, Brandenburg R, and Bruggeman P 2013 J. Phys. D: Appl. Phys. 48(17) 175203.

[9] Gershman S, Mozgina O, Belkind A, Becker K, and Kunhardt E 2007 Contributions to Plasma Physics 47(1-2) 19-25.

[10] Tachibana K, Takekata Y, Mizumoto Y, Motomura H, and Jinno M 2011 Plasma Sources Sci. Technol. 20(3) 034005.

[11] Bruggeman P, Degroote J, Vierendeels J, and Leys C 2008 Plasma Sources Sci. Technol. 17(2) 025008 .

[12] Babaeva N and Kushner M 2009 J. Phys. D: Appl. Phys. 42(13) 132003.

[13] Tian W, Tachibana K and Kushner M 2014 J. Phys. D: Appl. Phys. 47(5) 055202.

[14] Levko D, Sharma A, and Raja L L 2016 J. Phys. D: Appl. Phys., 49(28), 285205.

[15] Shih K and Locke B 2011 IEEE Trans Plasma Sci. 39(3) 883-892.

[16] Sunka P, Babický V, Clupek M, Lukes P, Simek M, Schmidt J, and Cernak M 1999 Plasma Sources Sci. Technol. 8(2) 258-265.

[17] Bruggeman P, Schram D, González M Á, Rego R, Kong M G, and Leys C 2009 Plasma Sources Sci. Technol. 18(2) 025017.

[18] Shih K and Locke B 2009 Plasma Chemistry and Plasma Processing. 30(1) 1-20.

[19] Thagard S M, Prieto G, Takashima K, and Mizuno A 2012 IEEE Trans. Plasma Sci. 40 2106-11. 
[20] Hamdan A and Cha M S 2016 IEEE Trans Plasma Sci. 44(12) 2988-2994.

[21] Hamdan A and Cha M S 2016 J. Phys. D: Appl. Phys. 49(24) 245203.

[22] Hamdan A and Cha M S 2015 J. Phys. D: Appl. Phys. 48(40) 405206.

[23] Starikovskiy A 2013 Plasma Sources Sci. Technol. 22(1) 012001.

[24] Vanraes P, Nikiforov A, and Leys C 2012 J. Phys. D: Appl. Phys. 45(24) 245206.

[25] Hayashi Y, Takada N, Kanda H, and Goto M 2015 Plasma Sources Sci. Technol. 24(5) 055023. 


\section{FIGURES CAPTIONS}

Figure 1. The experimental setup a) for imaging and electrical characterization and b) for optical emission spectroscopy.

Figure 2. Variation of the discharge probability in water discharge as a function of EC for $d=1$ and $2.5 \mathrm{~mm}$ at $V_{a}=15 \mathrm{kV}$.

Figure 3. Discharge probability as a function of electric conductivity in water $(\varepsilon=80)$ and methanol $(\varepsilon=30)$ with $d=2.5 \mathrm{~mm}$ applying $V_{a}=15 \mathrm{kV}$.

Figure 4. Current profiles of the misignited discharges for methanol (a) and water (b) solutions of various ECs with $d=2.5 \mathrm{~mm}$ applying $V_{a}=15 \mathrm{kV}$.

Figure 5. Images (exposure time $=1 \mu \mathrm{s}$ ) of discharges for various EC in water and methanol with $d=1 \mathrm{~mm}$ applying $V_{a}=15 \mathrm{kV}$.

Figure 6. The variation of the emission volume as a function of EC of water and methanol with d $=1 \mathrm{~mm}$ and $V_{a}=15 \mathrm{kV}$.

Figure 7. Lifetime of the plasma emission with increasing EC of water and methanol with $V_{a}=$ $15 \mathrm{kV}$ and for $\mathrm{d}=1$ and $2.5 \mathrm{~mm}$.

Figure 8. The variation of the $Q_{\text {injected }}$ as a function of EC of in-water and in-methanol discharges with $V_{a}=15 \mathrm{kV}$ and for $d=1$ and $2.5 \mathrm{~mm}$.

Figure 9. Emission spectra obtained by discharging in methanol $(E C=0.01,0.9$, and 1.5 $\mathrm{mS} / \mathrm{cm})$ and in water $(E C=0.01 \mathrm{mS} / \mathrm{cm})$ with $d=1 \mathrm{~mm}$ and $V_{a}=15 \mathrm{kV}$; the exposure time is 1 $\mu$ s. The insert is a zoom in 520-630 $\mathrm{nm}$ region showing the second order of $W_{I}$ (black) and $W_{I I}$ (red) lines.

Figure 10. Time evolution of Ha lines observed by discharging in a) water and b) methanol with $d=2.5 \mathrm{~mm}, V_{a}=15 \mathrm{kV}$, and EC of $10 \mu \mathrm{S} / \mathrm{cm}$; the exposure time is $100 \mathrm{~ns}$. c) Time evolution of $n_{e}$ for water and methanol and for $d=1$ and $2.5 \mathrm{~mm}$ at $V_{a}=15 \mathrm{kV}$.

Figure 11. a) Numerical simulations of electric field for $d=1 \mathrm{~mm}$, bubble diameter $=1 \mathrm{~mm}$, and $V_{a}=15 \mathrm{kV}$ in water $(\varepsilon=80)$. b) Zoom of the square drawn in a) and we show the anode tip and the anode-side of the bubble. The background is for $\varepsilon=80$ while the profiles along the bubble axis are for $\varepsilon=80$ (solid line) and 30 (dashed line).

Figure 12. a) Variation of the intensity of electric field at the anode and the anode-side bubble as a function of $\varepsilon$ for anode-bubble distance of a) $1 \mu \mathrm{m}, \mathrm{b}) 5 \mu \mathrm{m}$, and c) $10 \mu \mathrm{m} . \mathrm{d}=1 \mathrm{~mm}$, bubble diameter $=1 \mathrm{~mm}$, and $V_{a}=15 \mathrm{kV}$. 